\title{
Research Square \\ Determinant of Early Age Sexual Initiation: Associated Factors Among Rural Women in Ethiopia: Evidence From EDHS 2016.
}

Solomon Sisay Mulugeta ( $\sim$ solsisay23@gmail.com )

Debre Tabor University

Shewayiref Geremew Gebremichael

Debre Tabor University

\section{Research}

Keywords: Early age sexual initiation, EDHS, HIV/AIDS, Women, Rural Ethiopia

Posted Date: June 8th, 2021

DOI: https://doi.org/10.21203/rs.3.rs-578274/v1

License: (c) (1) This work is licensed under a Creative Commons Attribution 4.0 International License.

Read Full License 


\title{
Determinant of Early age sexual initiation: The Associated factors among rural women in Ethiopia: Evidence from EDHS 2016
}

\author{
Solomon Sisay Mulugeta ${ }^{1 *}$, Shewayiref Geremew Gebremichael ${ }^{1}$ \\ ${ }^{1}$ Department of Statistics, Debre Tabor University, Debre Tabor, Amhara, Ethiopia \\ *Correspondence: solsisay23@gmail.com
}




\section{Plain English summary}

Early age sexual initiation exposes to a high rate of reproductive health complications such as HIV infection, sexually transmitted infections, unwanted pregnancy, and pregnancy-related complications. Girls are at a much greater risk at early ages, because of both biological and sociocultural factors.

This study contains the Ethiopian Demographic and Health Survey (EDHS) 2016 women dataset. A total of 8001 women of under reproductive age (15-49 years) those who had at least one set of sexual intercourse, were involved in the analysis.

Among the respondents starting the first sex more than half 4772 of rural women was experienced sexual intercourse at early age. The higher proportion of experiencing early age sexual initiation among rural women was found in Amhara (18.63\%) region.

The most important variable such as: Age of women, geopolitical region, women age at first birth, HIV/AIDS awareness, chat chewing, willingness at first sex, marital status, and educational attainment were observed as statistically significant factors.

In conclusion: The commencement of early age sexual initiation among reproductive-age women was high. These studies have suggested that continued programmatic and policy initiatives should be directed to improve adolescent's overall situation as a means of improving their sexual health. 


\begin{abstract}
Background: The aim of this study was to examine the determinant factors on early age sexual intercourse among rural women in Ethiopia.
\end{abstract}

Methods: This study contains further analysis of the Ethiopian Demographic and Health Survey (EDHS) 2016 dataset. A total of 8001 participants of under reproductive age (15-49 years) women, who had at least one set of sexual intercourse, were involved in the analysis. The binary logistic multivariable analysis result was obtained by STATA-14 software.

Results: Among the rural women in Ethiopia, more than half (59.6\%) of women was experienced sexual intercourse at early age. The higher proportion of experiencing early age sexual initiation among rural women was found in Amhara (18.63\%) region. Age of women [(OR= 0.374;95\%CI:0.301-0.465) of 20-24 years, $(\mathrm{OR}=0.44 ; 95 \% \mathrm{CI}: 0.36-0.54)$ of 25-49 years], geopolitical region [Amhara $(\mathrm{OR}=1.514 ; 95 \% \mathrm{CI}: 1.25-1.83)$, Oromia (OR=0.74; 95\%CI:0.640.905), Somali(OR=0.47; 95\% CI: 0.364-0.658), SNNPR (OR=0.5; 95\% CI: 1.13-1.81), and Dire Dawa (OR=0.68; 95\% CI: 0.49-0.941)]; women age at first birth (OR=0.3; 95\% CI: 0.11-0.5) of greater than 18 years old; HIV/AIDS awareness (OR=0.4; 95\% CI: 0.183-1.604, had awareness); chat chewing $(\mathrm{OR}=1.23$; 95\% CI:1.03-1.47); willingness at first sex (OR=2.51; 95\% CI: 1.22-3.8, by force); marital status [married women (OR=2.52; 95\% CI:1.69-3.75); divorced/separated women $(\mathrm{OR}=2.39 ; 95 \% \mathrm{CI}: 1.58-3.63)]$; and educational attainment [secondary school and above completed $(\mathrm{OR}=0.231$; 95\% CI: $0.183-0.291)$ and primary level of education $(\mathrm{OR}=0.67 ; 95 \% \mathrm{CI}$ : 0.06-0.76)] were examined as statistically significant factors.

Conclusion: The commencement of early age sexual initiation among reproductive-age women was high. This study suggests that continued programmatic and policy initiatives should be directed to improve adolescent's overall situation as a means of improving their sexual health.

Keywords: Early age sexual initiation, EDHS, HIV/AIDS, Women, Rural Ethiopia 


\section{Introduction}

Sexual initiation is any known attempt on the part of the respondent to establish sexual contact. Sexual initiation is likely to be related to an adolescent's perception of their transition to adulthood. Worldwide, the average age of sexual initiation among adolescents is 16-18years. However, some studies have reported a decreasing trend in AFSI (Average age of first sexual (vaginal) intercourse) among adolescents around the world. Early-onset of sexual activity has been associated, to some extent, with multiple sexual partners, infrequent use of condoms, unplanned pregnancy, unsafe abortion, and sexually transmitted disease and human immune-deficiency virus infection $[1,2]$.

Adolescents attempt to detach themselves from the infantile phase to assume behaviors that transform them into socially accepted adults. In this process of transformation, they become more aware of their sexuality, which has been constructed throughout life through the interpersonal relationships established between the individual and the environment in which they live as an opportunity to develop their sexual interaction capacity. However, while the development of sexuality is something natural and expected, young people have increasingly engaged in premature and risky sexual activities $[3,4]$.

For young people who start their sexual life in adolescence age, this event is perceived as an opportunity to express the exercise of autonomy and sexual freedom, sensations that evidence a more emotional than rational meaning. However, many adolescents do not understand that this event also represents their most intense introduction in the group vulnerable to Sexually Transmitted Infections (STIs), unplanned pregnancy, and abortion[5]. Since, they do not perceive unsafe sexual practice risks, some adolescents' experience this moment without worrying about the possible negative consequences that this event may cause. There is an ongoing debate regarding the nature of gender differences in sexual problems. One feature of this debate centers on the extent to which biological, psychosocial, and cultural factors each contribute to these various difficulties[6].

Early initiation of sexual activity is linked to higher numbers of non-marital sex partners, minimal condom use, increased rates of STIs, increased rates of out of marriage pregnancy and giving birth, increased single parenthood, decreased marital stability, increased maternal and childhood 
poverty, increased abortion, increased depression, and reduced happiness. These consequences incur a high cost to society[7].

Worldwide, early sexual initiation exposes to a high rate of reproductive health complications such as HIV infection, sexually transmitted infections, unwanted pregnancy, and pregnancy-related complications. During this age, pregnancy-related complications are aggravated by physiological immaturity. Studies have shown that earlier sexual engagement is related to the higher risk of acquiring STIs and getting pregnant compared to adults. Early sexual engagement may also hinder international initiatives such as reducing maternal and child mortality and morbidity, universal education for all, and reduction of poverty by promoting a high number of early child bearings coupled with larger population growth[8].

Girls are at a much greater risk at early ages because of both biological and socio-cultural factors. Young girls in Ethiopia are more vulnerable to HIV than boys because of early age at sexual debut, early marriage, sexual abuse, and violence, such as rape and abduction. Sexual mixing patterns are more important than the age at sexual debut in putting girls at higher risk of HIV than boys. As well, adolescent girls are at risk because they are unlikely to have had any training or experience in sexual negotiation skills, and are especially vulnerable in situations with older men where age, wealth, physical strength, and other power dynamics put them in a disadvantaged group [9].

Early sexual initiation is among the most contributors to high population growth in Ethiopia. Despite the definition of sexual initiation being differently used in different kinds of literature, in Ethiopia, early sexual initiation is defined as experiencing sexual intercourse before the age of 18 years. Practicing sexual intercourse before the age of 18 years has a lot of health problems such as getting infected with human immune deficiency virus, unwanted pregnancy, other sexually transmitted diseases, and fistula; because girls before the age of 18 years may not be physiologically ready to practice sexual intercourse and give birth. Even though the health policy of Ethiopia gives due attention to youth reproductive health, problems related to early sexual initiation are still very high[10].

Despite the factors associated with early age sexual initiation and the reasons, adolescents begin sexual intercourse, geographical variability of sexual initiation and identification of risk factors are not well studied among rural women in Ethiopia. Thus, the main contribution of this study would be to examine the most significant predictors of early-age sexual initiation. 


\section{Methods}

\section{Sources of Data}

This study used data from Ethiopia Demographic and Health Survey (EDHS), 2016. It was implemented by the Central Statistical Agency (CSA) from January 18, 2016, to June 27, 2016. The survey is usually conducted at a five-year interval in a country. The country has undertaken four consecutive DHS surveys in (2000, 2005, 2011, and 2016).

\section{Sampling Techniques}

A stratified two-stage cluster sampling technique using a nationally representative populationbased survey was employed. The data were collected from 645 enumeration areas (EAs) (202 urban and 443 rural areas) independently in each stratum of the two stages using systematic sampling with probability proportional to size. This study was used Statistical Package for Social Science (SPSS) version 21 for data extraction, and STATA version 14 as a tool for analysis. STATA was used in running the binary logistic regression and statistical analysis. SPSS was employed to extract and sorting of data.

\section{Sample Size and Variables}

After applying the weighting technique, a total of 8001 rural women of reproductive age (15-49 years) who had at least one occurrence of sexual intercourse was retrieved and included for the analysis. The response variable was early-age sexual initiation, categorized dichotomously as (Yes, No). Women, who were engaged in sexual intercourse before the age of 16.6, were categorized as "Yes" and those who didn't engage as "No". The value of 16.6 was selected as it was the median cut-point age of engaging in sexual intercourse, nationally. Descriptive statistics and multivariable binary logistic regression analyses have been used in this study.

\section{Binary Logistic Regression}

Logistic regression is part of a family of models called the Generalized Linear Model, used when the response variable is qualitative or categorical and the regressors can be continuous and/or categorical [11].

Binary logistic regression techniques resolve inconsistencies associated with dichotomous dependent data and the assumptions of linear regression methods. The independent variables that 
are used for outcome prediction may be dichotomous, categorical, or continuous. Binary logistic regression is commonly used in manufacturing and health-related studies. It can be used for any application where binary outcomes can be predicted. Logistic regression is based on the logit transformation of the dependent variable. The logit transformation generates a continuous logarithmic curve from non-continuous data so that a regression model can be developed. The outcome probabilities for each dependent variable value are the basis for the model. The logit transformation is necessary since dichotomous dependent data violates ordinary least squares assumptions. Another issue with dichotomous data is that the error terms are not normally distributed, thus the ordinary sum of squares of regression and all normality tests are invalid [12]. Logistic regression has two main uses: the first is, the prediction of a group membership. Since logistic regression calculates the probability of success over the probability of failure; the results of the analysis are in the form of an odds ratio. Logistic regression also provides knowledge of the relationships and strengths among the variables. There are two primary reasons for choosing the logistic distribution function. First, from a mathematical point of view, it is an extremely flexible and easily used function, and second, it lends itself to a clinically meaningful interpretation [11].

Logistic regressions work with odds and odds ratios. The odds are simply the ratio of the probabilities for the two possible outcomes. If ' $\mathrm{P}$ ' is the probability that the event will occur, then ' $1-\mathrm{P}$ ' is the probability that the event will not occur:

$$
O d d=\frac{P}{1-P}
$$

In $2 \times 2$ contingency table, within row 1 the odds of success are $\operatorname{odds} 1=\frac{P 1}{1-P 1}$, and within row 2 the odds of success equal $O d d s 2=\frac{P 2}{1-P 2}$. The ratio of the odds from the two rows, $\theta=\frac{\text { odds } 1}{\text { odds } 2}=\frac{\frac{P 1}{1-P 1}}{\frac{P 2}{1-P 2}}=\frac{P 1(1-P 2)}{P 2(1-P 1)}$ is the odds ratio.

When the response variable is binary, there is considerable empirical evidence that the shape of the response function should be non-linear. A monotonically increasing or decreasing S-shaped or reverse S-shaped function.

Maximum likelihood (ML) estimation is the most common method used to calculate the logit coefficients. The maximum likelihood estimates are the values of ' $\beta$ ' that maximize the above loglikelihood function. Through maximization of the log-likelihood function, we can theoretically 
estimate the vector of parameter ' $\beta$ '. However, the equation is non-linear in ' $\beta$ ', and as a result, the estimates do not have a closed-form expression. Therefore, ' $\beta$ ' will be obtained by maximizing log-likelihood using the iterative algorithm method [11].

The Goodness of fit of the model: the goodness of fit or calibration of a model measures how well the model describes the response variable. Assessing goodness of fit involves investigating how the predicted values are closer to the observed values.

The Hosmer-Lemeshow (H-L) test: a better way of assessing the fit of a logistic regression model is comparing the observed and expected numbers of positives for different subgroups of the data. The Hosmer-Lemeshow goodness of fit test is useful for assessing overall model fit, particularly when we have many predictor variables, or some of the predictor variables are continuous. The test is similar to an $x^{2}$ goodness of fit test and has the advantage of partitioning the observations into groups of approximately equal size, and therefore, there are less likely to be grouped with very low observed and expected frequencies.

If the p-value of the Hosmer-Lemeshow goodness-of-fit test is greater than 0.05, we will not reject the null hypothesis indicates there is no difference between observed and model-predicted values, implying that the model estimates are adequate to fit the data at an acceptable level or if the observed and expected numbers are sufficiently close, then we can assume that we have an adequate model.

\section{Result}

From (Table 1) evidence, 8001 rural women were included in this study. Among these 3229 (40.4\%) women were not engaged in sexual intercourse at an early age. Among Ethiopian rural women included in this study more than half 4772(59.6\%), women were had sexual intercourse at an early age or reported first sex at age 16.6 or under.

Table 1: Percentage of early age sexual initiation among rural women in Ethiopia, EDHS $2016(n=8001)$

\begin{tabular}{lrll}
\hline & Age group & Frequency & Percentage \\
\cline { 2 - 4 } Age at first sex & $\leq 16.6$ & 4772 & 59.6 \\
& $>16.6$ & 3229 & 40.4 \\
\hline
\end{tabular}


The higher proportion of experiencing early age sexual initiation among rural women was found in Amhara (18.63\%), Tigray (12.95\%), Afar (11.59\%), Gambela (7.4\%), and Harari (4.3\%).

(Figure 1)

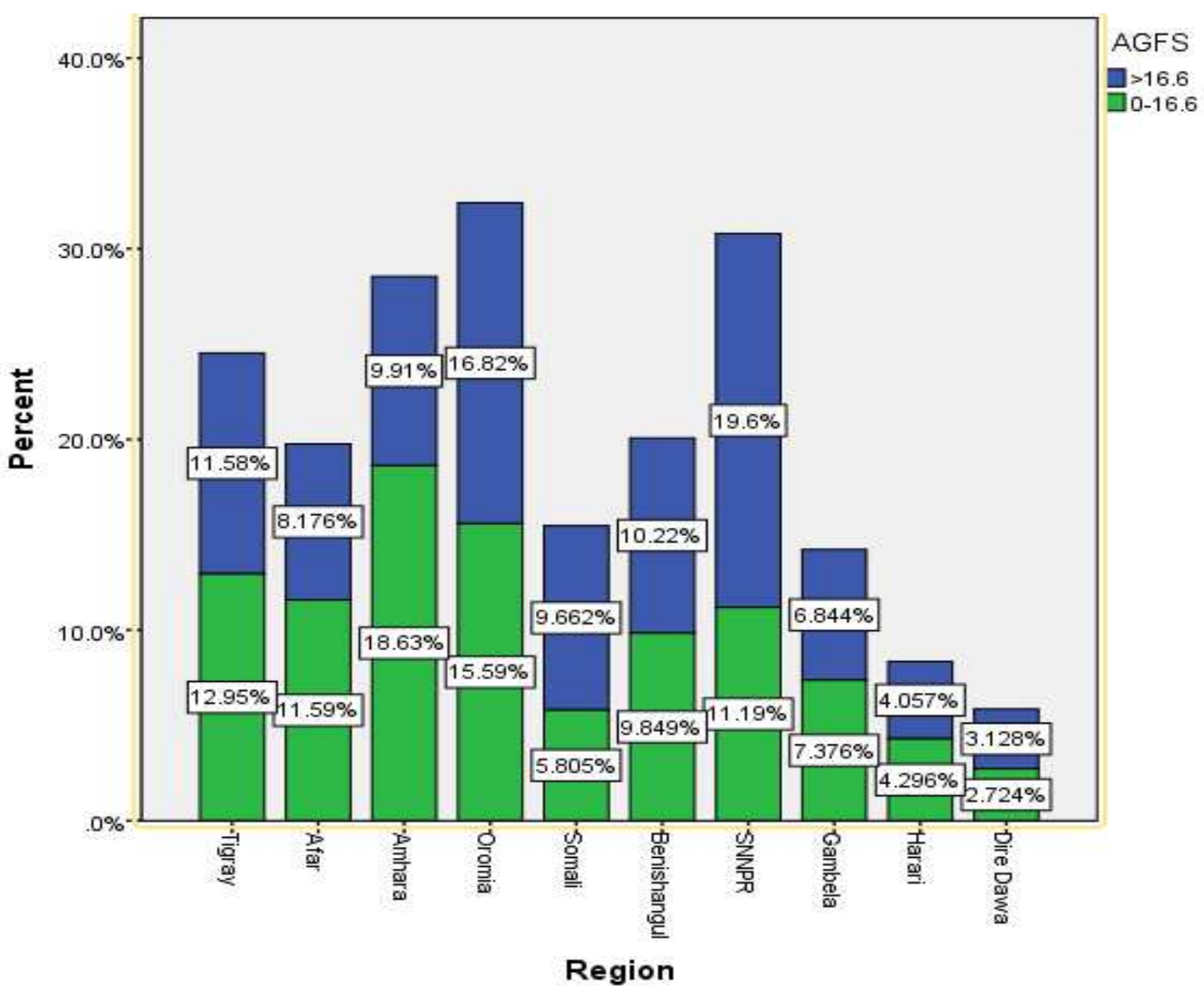

Figure 1: Geographical region on early age sexual initiation in rural Ethiopia, EDHS 2016 $(\mathbf{n}=\mathbf{8 0 0 1})$

From the total of 8001 women who had participated in this study, 4139(86.7\%) married women were experiencing early age sexual intercourse, and 53(1.1\%) were never married. Of the total respondents, 4723(99\%) women were participating in early age sexual intercourse at first sex were on her want, and 49(1\%) were engaged in forced. Moreover, almost all 4726(99\%) rural women participating in early age sexual intercourse were non-smokers. About 4288(89.9\%) of rural women participating in early age sexual intercourse were having awareness of HIV/AIDS. Of the respondents who are experiencing early age sexual intercourse, $4230(88.6 \%)$ of rural women were not participating in chat chewing, and 3176(66.6\%) were not drinking alcohol. Similarly, nearly three-fourths of the rural women $3454(72.4 \%)$ were non-educated and 141(3\%) were had 
secondary and above educational level. Among the rural women experiencing early age sexual initiation, 2808(58.8\%), 870(18.2\%), and 1094(22.9\%) were poor, middle, and rich economic status.

The chi-square test value is computed as the sum of the square of the differences between the observed and expected categorical responses and divided by the expected value, between two or more independent categories; it implies that the larger the value, the larger the difference, and the smaller the value, the smaller the difference. A P-value $\leq 0.05$ is used to test the presence of the significant association between the dependent and independent variables. The variable marital status $\left(x^{2}=15.4, \mathrm{p} \leq 0.001\right)$, women feeling in the first $\operatorname{sex}\left(x^{2}=4.4, \mathrm{p}=0.036\right)$, smoking cigarette $\left(x^{2}=5.1, \mathrm{p}=0.032\right)$, women HIV/AIDS awareness $\left(x^{2}=18.124, \mathrm{p} \leq 0.001\right)$, chewing $\operatorname{chat}\left(x^{2}=8.21, \mathrm{p}=0.004\right)$, drinking alcohol $\left(x^{2}=31.8, \mathrm{p} \leq 0.001\right)$, a women education level $\left(x^{2}=171.43, \mathrm{p} \leq 0.001\right)$, and wealth index $\left(x^{2}=4.76, \mathrm{p}=0.03\right)$ are statistically significant, this result implies that those variable are significantly associated with early age sexual initiation among women in Ethiopia.( Table 2)

Table 2: Summary statistics for the variables, EDHS 2016(n = 8001)

Variable Categories $\quad$ Age at first sex $\quad x^{2} \quad$ P-value
(in number and percentage)

\begin{tabular}{|c|c|c|c|c|c|}
\hline & & $>16.6$ & $\leq 16.6$ & & \\
\hline \multirow[t]{3}{*}{ Marital status } & Never married & $70(2.2 \%)$ & $53(1.1 \%)$ & 15.4 & 0.001 \\
\hline & Married & $2796(86.6 \%)$ & $4139(86.7 \%)$ & & \\
\hline & Divorced/separated & $363(11.2 \%)$ & $580(12.2 \%$ & & \\
\hline \multirow[t]{2}{*}{ First sex } & Wanted & $3210(99.4 \%)$ & $4723(99 \%)$ & 4.4 & 0.036 \\
\hline & Forced & $19(0.6 \%)$ & $49(1 \%)$ & & \\
\hline \multirow[t]{2}{*}{ Smoking } & No & $3190(98.8 \%)$ & $4726(99 \%)$ & 5.1 & 0.032 \\
\hline & Yes & $39(1.2 \%)$ & $46(1 \%)$ & & \\
\hline \multirow[t]{2}{*}{ HIV awareness } & No & $427(13.2 \%)$ & $484(10.1 \%)$ & 18.124 & 0.001 \\
\hline & Yes & $2802(86.8 \%)$ & $4288(89.9 \%)$ & & \\
\hline Chewing chat & No & $2927(90.6 \%)$ & $4230(88.6 \%)$ & 8.21 & 0.004 \\
\hline
\end{tabular}


Yes

Drinking No

alcohol

Yes

Women No education

Education

level

Secondary and above

Wealth index Poor

Middle

Rich
$302(9.4 \%) \quad 542(11.4 \%)$

$2341(72.5 \%) \quad 3176(66.6 \%) \quad 31.8$

0.001

$888(27.5 \%) \quad 1596(33.4 \%)$

$2027(62.8 \%) \quad 3454(72.4 \%) \quad 171.43 \quad 0.001$

905(28\%) 1177(24.7\%)

297(9.2\%) 141(3\%)

$\begin{array}{llll}1825(56.5 \%) & 2808(58.8 \%) & 4.76 & 0.03\end{array}$

$605(18.7 \%) \quad 870(18.2 \%)$

799(24.7\%) 1094(22.9\%)

The Hosmer-Lemeshow test shows that $x^{2}=1.722$ with a $p$-value of 0.988 . Therefore, at a $5 \%$ level of significance, we do not reject the null hypothesis that the predicted and observed values are closed and concluded that the model is adequate. (Table 3)

Table 3: Hosmer-Lemeshow Test

\begin{tabular}{llll}
\hline Step & Chi-square & Df & Sign \\
\hline 1 & 1.722 & 8 & 0.988 \\
\hline
\end{tabular}

Table 4: Multivariable analysis on women early age sexual initiation among rural women in Ethiopia, EDHS $2016(n=8001)$

\begin{tabular}{lccccc}
\hline Variables & $\begin{array}{l}\text { Odds } \\
\text { ratio }\end{array}$ & Std.err & $\mathrm{Z}$-value & $\mathrm{P}>|\mathrm{Z}|$ & {$[95 \% \mathrm{CI}]$} \\
\hline Age of women & & & & & \\
15-19 years & $\mathbf{1 . 0}$ & & & & \\
20-24 years & 0.37 & 0.042 & -8.84 & $0.001^{*}$ & $0.301,0.465$ \\
25-49 years & 0.44 & 0.047 & -7.74 & $0.001^{*}$ & $0.360,0.540$ \\
Region & & & & & \\
Tigray & $\mathbf{1 . 0}$ & & & & \\
Afar & 0.99 & 0.130 & -0.05 & 0.96 & $0.773,1.280$ \\
\hline
\end{tabular}




\begin{tabular}{lccccc}
\hline Amhara & 1.51 & 0.150 & 4.30 & $0.001^{*}$ & $1.253,1.830$ \\
Oromia & 0.74 & 0.080 & -2.93 & $0.003^{*}$ & $0.604,0.905$ \\
Somali & 0.47 & 0.064 & -5.51 & $0.001^{*}$ & $0.364,0.618$ \\
Benishangul & 0.84 & 0.091 & -1.58 & 0.115 & $0.683,0.104$ \\
SNNPR & 0.49 & 0.051 & -6.86 & $0.001^{*}$ & $0.410,0.610$ \\
Gambela & 1.19 & 0.146 & 1.47 & 0.141 & $0.940,1.520$ \\
Harari & 0.82 & 0.013 & -1.30 & 0.194 & $0.604,1.110$ \\
Dire Dawa & 0.68 & 0.114 & -2.32 & $0.020^{*}$ & $0.490,0.941$ \\
Religion & & & & & \\
Orthodox & $\mathbf{1 . 0}$ & & & & \\
Muslim & 1.04 & 0.090 & 0.52 & 0.601 & $0.890,1.230$ \\
Other & 0.82 & 0.160 & -1.04 & 0.293 & $0.570,1.190$ \\
Cigarette smoking & & & & & \\
No & & & & & \\
Yes & $\mathbf{1 . 0}$ & & & & \\
Number of sexual \\
partners
\end{tabular}




\begin{tabular}{llllll}
\hline Greater than 18 & 0.30 & 0.093 & -3.41 & $0.001 *$ & $0.110,0.500$
\end{tabular}

\section{First sex}

Wanted

\section{0}

Forced

$$
2.5
$$

0.620

2.65

$0.008 * 1.220,3.800$

Wealth index

Poorest

\section{0}

Middle

$$
0.94
$$

0.630

$-0.94$

0.350

$0.824,1.071$

Richest

1.05

0.007

0.70

$0.500 \quad 0.923,1.190$

Marital status

Never married

\section{0}

Married

2.52

0.512

4.53

$0.001 * \quad 1.690,3.750$

Other

2.39

0.508

4.11

$0.001 * \quad 1.580,3.630$

Educational level

Illiterate $\quad \mathbf{1 . 0}$

\begin{tabular}{llllll} 
Primary & 0.67 & 0.004 & -6.63 & $0.001 *$ & $0.0598,0.76$ \\
Secondary and above & 0.23 & 0.027 & -12.44 & $0.001 *$ & $0.183,0.291$ \\
Constant & 1.32 & 0.323 & 1.14 & 0.254 & $0.820,2.132$ \\
\hline
\end{tabular}

Table 4 shows the effect of explanatory variables on a dependent variable. The result of the multivariable analyses on binary logistic regression revealed that the factors such as regions: Amhara (OR=1.514; 95\%CI:1.25-1.83), Oromia (OR=0.74; 95\%CI:0.64-0.905), Somali $(\mathrm{OR}=0.47 ; 95 \% \mathrm{CI}: 0.364-0.658), \mathrm{SNNPR}(\mathrm{OR}=0.5 ; 95 \% \mathrm{CI}: 1.13-1.81)$, and Dire Dawa $(\mathrm{OR}=0.68$; 95\% CI: 0.49-0.941) show a significant effect of early age sexual initiation on rural women in Ethiopia compared to Tigray region. Similarly, women age group $(\mathrm{OR}=$ 0.374;95\%CI:0.301-0.465) of 20-24 years, $(\mathrm{OR}=0.44 ; 95 \% \mathrm{CI}: 0.36-0.54)$ women age group between 25-49 years were significant factors of early age sexual initiation.

Women reside in rural had educational level of secondary school and above completed $(\mathrm{OR}=$ 0.231; 95\% CI: 0.183-0.291) and primary level of education (OR=0.67; 95\% CI: 0.06-0.76) and had HIV awareness (OR=0.4; 95\% CI: 0.183-1.604); chewing chat(OR=1.23; 95\% CI:1.03-1.47); 
age at first birth of greater than 18 years $(\mathrm{OR}=0.3 ; 95 \% \mathrm{CI}$ : $0.11-0.5)$; women started first sex was by force $(\mathrm{OR}=2.51 ; 95 \% \mathrm{CI}$ : $1.22-3.8)$ were significant factors of early age sexual initiation.

Regarding the marital status of women in rural Ethiopia: married women (OR=2.52; 95\% CI: 1.69$3.75)$; divorced/separated women $(\mathrm{OR}=2.39$; 95\% CI: 1.58-3.63) were significant factors of early age sexual initiation compared to the never-married women.

\section{Discussions}

A Binary Logistic Regression Analysis is used to identify the most important determinant factors which are associated with early age sexual initiation of rural women in Ethiopia. The age of first sexual initiation (16.6) in this study is slightly advanced than previous studies done in Northeast Ethiopia (16.8 years) [13], in Northwest Ethiopia (15 years) [14], in Northwest Ethiopia (13.5 years) [15], and in southern Ethiopia (16 years) [16]. The difference may be happened by the cutoff point for age at coital debut was based on the median age(16.6) values and the median age of marriage (17.1) value of Ethiopian women included in the study which was the main reason for early sexual initiation in rural women[13].

The odds of women aged between, 20-24 and 25-49 years were 0.374 and 0.44 times less likely to participate in early age sexual intercourse compared to the age group 15-19 years for the rural women, holding the other variables constant. This finding is consistent with studies conducted in Ireland [14] and China [15]. The current study generally implies that early age sexual initiation was highest in adults age between 15 and 19 years old than in the other age groups. The main reason for early sexual initiation among women at adult age might be because of early marriage. It was also sophisticated in rural women.

According to this study, geographical variation was observed in the rural women's early age sexual initiation. It was found that the odds of early age sexual initiation in the Amhara region were 51.4\% more likely to participate in early age sexual initiation compared to the Tigray region and the odds of early age sexual initiation in Oromia, Somali, SNNPR and Dire Dawa were 0.74,0.74,0.496 and 0.68 times less likely to participate on early age sexual initiation compared to Tigray region, for the rural women, holding the other variables constant. This study finding is consistent with studies conducted in Ethiopia [16] and Nigeria[17]. 
According to the current finding, as compared with women those who have awareness about HIV/AIDS were 0.4 times less likely to be participated in early age sexual initiation than for women who have no awareness about HIV, holding the other variable constant. This finding is consistent with studies done in Ethiopia [18]. This might be justified as according to the awareness created for rural women about HIV/AIDS and sexually transmitted infection was being not much. Moreover, there was a significant association between chewing chat and early age sexual initiation. The odds of women who are chewing chat were $23 \%$ more likely to participate in early age sexual intercourse compared to non-chewing, holding the other variable constant. This finding is consistent with studies done in Ethiopia [19, 20]. It shows that drinking alcohol usually follows khat (chat) chewing and might be associated with unprotected sex and early initiation. The probable reason for this could be drug trafficking and drug abuse, although no problems in the past and now are becoming more common in Ethiopia.

Besides, the odds of women with an age of first birth greater than 18 years were 0.3 times less likely to participate in early age sexual intercourse compared to women with an age of first birth less than 18 years, holding the other variable constant. In general, this finding shows that women, whose age of first birth at teenage, were more likely to have early sexual debut than the other age group. This study is in line with the study done by $[17,21]$. This might be a possible reason that early sexual debut and childbearing were more common among the least educated and/or rural females. This was the consequence of early marriage.

According to the current study, women's first sex and early sexual initiation were significantly associated. The odds of women started her first sex by force was $51 \%$ more likely to participate in early age sexual intercourse compared to women who started their first sex by want. The current study implies that women who had coerced sexual debut were more likely to participate in early age sexual intercourse than women who had a sexual debut by went. This study was a confirmed study done in South Africa[22] and comparable to a local study[23]. The possible justification for this might be noted that public exclusion was associated with a significantly higher order of coerced first sex among women.

The result of this study revealed that another significant association was also held between the marital status of rural women and early age sexual initiation. The odds of being married and widowed, divorced/separated were 2.52 and 2.39 times more likely to experience early sexual 
initiation when compared to never-married respondents; respectively, holding the other variable constant. This finding is inlined to studies done in Ethiopia [16, 19, 24]. This might be occurred due to the rural women engaged in early marriage (before the age of 18 years old) which is the possible situation for women to be engaged in early age sexual intercourse activity. According to EDHS 2016 report, the median age of first marriage in the country was 17.1 years [13].

There was also held a strong statistical association between education and early sexual initiation. The odds of women with primary and secondary and above had 0.67 and 0.231 times less likely than the odds of women who experienced early age sexual initiation compared to women having no education; respectively holding the other variables constant. This finding was confirmed in previous studies [16, 25-27]. It shows that women with higher educational qualifications were significantly less likely to initiate at early age sexual intercourse. In general, this indicates that the higher the level of education, the less likely to participate in early age sexual initiation and they might save themselves from being involved in sex at an early age.

\section{Conclusion and Recommendation}

This study aimed to examine the various determinants of early age sexual intercourse among women in rural Ethiopia. Among the rural women in Ethiopia, more than half (59.6\%) of women were experienced sexual intercourse at an early age. Generally, the commencement of early age sexual initiation among reproductive-age women was high. And, early age sexual initiation was varied across the regions. Age of women, region, age at first birth, HIV/AIDS awareness, chat chewing, and willingness to first sex, marital status, and educational attainment was examined as statistically significant factors. Especially, traditional and cultural beliefs that seem to encourage early marriage and early birth lead to early age initiation of sexual intercourse may be the principal reasons for the observed geographical disparities. This study suggests that continued programmatic and policy initiatives should be directed to improve the adolescent's overall situation as a means of improving their sexual health. Strengthening the norm of virginity should be advocated to

minimize the commencement of early age sexual initiation among women. At the same time, the findings argue strongly for programmatic measures that delay marriage.

Therefore, the concerned body should create a suitable environment for women to discuss and learn about sexual and reproductive health issues and should identify and tackle behaviors that could inspire women for early sexual initiation. 


\section{Data sharing}

The data for this study were sourced from Demographic and Health Surveys (DHS) and are available at http://www.dhsprogram.com/data/available-datasets.cfm.

\section{References}

1. Busse, P., et al., The role of communication with friends in sexual initiation. Communication research, 2010. 37(2): p. 239-255.

2. Pettifor, A., et al., Early coital debut and associated HIV risk factors among young women and men in South Africa. International perspectives on sexual and reproductive health, 2009: p. 82-90.

3. Organization, W.H., World health statistics 2016: monitoring health for the SDGs sustainable development goals. 2016: World Health Organization.

4. $\quad$ UNAIDS, U., Prevention gap report. UNAIDS Geneva, 2016.

5. Stöckl, H., et al., Is early sexual debut a risk factor for HIV infection among women in subsaharan Africa? A systematic review. American Journal of Reproductive Immunology, 2013. 69: p. 27-40.

6. Ghebremichael, M., U. Larsen, and E. Paintsil, Association of age at first sex with HIV-1, HSV-2 and other sexual transmitted infections among women in northern Tanzania. Sexually transmitted diseases, 2009. 36(9): p. 570.

7. Caminis, A., et al., Psychosocial predictors of sexual initiation and high-risk sexual behaviors in early adolescence. Child and adolescent psychiatry and mental health, 2007. 1(1): p. 14.

8. Zimmer-Gembeck, M.J. and M. Helfand, Ten years of longitudinal research on US adolescent sexual behavior: Developmental correlates of sexual intercourse, and the importance of age, gender and ethnic background. Developmental review, 2008. 28(2): p. 153-224.

9. Berhane, Y., et al., HIV/AIDS in Ethiopia: an epidemiological synthesis, 2008, The World Bank.

10. Ambaw, F., A. Mossie, and T. Gobena, Boy/girl friend and virginity values, and stigma related to condom among Jimma University students. Ethiopian journal of health sciences, 2010. 20(3).

11. Hosmer Jr, D.W., S. Lemeshow, and R.X. Sturdivant, Applied logistic regression. Vol. 398. 2013: John Wiley \& Sons.

12. Healy, L.M., Logistic regression: An overview. Eastern Michighan College of Technology, 2006.

13. Csa, I., Central statistical agency (CSA)[Ethiopia] and ICF. Ethiopia demographic and health survey, Addis Ababa, Ethiopia and Calverton, Maryland, USA, 2016.

14. Young, H., L. Burke, and S.N. Gabhainn, Sexual intercourse, age of initiation and contraception among adolescents in Ireland: findings from the Health Behaviour in School-aged Children (HBSC) Ireland study. BMC Public Health, 2018. 18(1): p. 362. 
15. Shu, C., et al., Association between age at first sexual intercourse and knowledge, attitudes and practices regarding reproductive health and unplanned pregnancy: a cross-sectional study. Public health, 2016. 135: p. 104-113.

16. Nigatu, A.M., A.Y. Birhanu, and B.F. Endehabtu, Geographical variations of early age sexual initiation among reproductive-age women in Ethiopia: evidence from EDHS 2016. Archives of Public Health, 2020. 78(1): p. 1-12.

17. Uthman, O.A., Geographical variations and contextual effects on age of initiation of sexual intercourse among women in Nigeria: a multilevel and spatial analysis. International Journal of Health Geographics, 2008. 7(1): p. 27.

18. Anwar, M., et al., Awareness of school students on sexually transmitted infections (STIs) and their sexual behavior: a cross-sectional study conducted in Pulau Pinang, Malaysia. BMC public health, 2010. 10(1): p. 47.

19. Mazengia, F. and A. Worku, Age at sexual initiation and factors associated with it among youths in North East Ethiopia. Ethiopian Journal of Health Development, 2009. 23(2).

20. Molla, M., Y. Berhane, and B. Lindtøøn, Traditional values of virginity and sexual behaviour in rural Ethiopian youth: results from a cross-sectional study. BMC public health, 2008. 8(1): p. 9.

21. Doyle, A.M., et al., The sexual behaviour of adolescents in sub-Saharan Africa: patterns and trends from national surveys. Tropical Medicine \& International Health, 2012. 17(7): p. 796-807.

22. Richter, L., et al., Early sexual debut: Voluntary or coerced? Evidence from longitudinal data in South Africa-the Birth to Twenty Plus study. South African medical journal, 2015. 105(4): p. 304-307.

23. Erulkar, A. and A. Ferede, Social exclusion and early or unwanted sexual initiation among poor urban females in Ethiopia. International perspectives on sexual and reproductive health, 2009: p. 186-193.

24. Digafe, T.N., Determinants of sexual debut among high school girl students in Arsi Zone, Huruta town, Ethiopia. Epidemiology: Open Access, 2017. 7(3).

25. Wheeler, S.B., Effects of self-esteem and academic performance on adolescent decisionmaking: an examination of early sexual intercourse and illegal substance use. Journal of Adolescent Health, 2010. 47(6): p. 582-590.

26. Okigbo, C.C. and I.S. Speizer, Determinants of sexual activity and pregnancy among unmarried young women in urban Kenya: a cross-sectional study. PLoS One, 2015. 10(6): p. e0129286.

27. Nigatu, D.T., et al., Sexual initiation and associated factors among young women in West Shoa, Ambo Town, Ethiopia: a community-based cross-sectional study. BMC women's health, 2018. 18(1): p. 76.

\section{Author information}

\section{Affiliations}

Department of Statistics, Debre Tabor University, Debre Tabor, Ethiopia

Solomon Sisay Mulugeta, Shewayiref Geremew Gebremichael, 


\section{Corresponding author}

Correspondence: solsisay23@gmail.com.

\section{Additional information}

\section{Competing financial interests}

The authors declare no competing financial interests exist.

\section{Authors' contribution}

SS carried out the data extraction, designed the study, performed analysis and interpretation, and draft the manuscript. SG designed the study, participated in the analysis and interpretation of the drafted manuscript. Both authors read and approved the final manuscript.

\section{Rights and permissions}

\section{Ethics approval and consent to participate}

Written consent was obtained from the Measure DHS International Program which authorized the data-sets. The data used in this study were publicly available, aggregated secondary data with not having any personal identifying information that can be linked to particular individuals, communities, or study participants. Confidentiality of data maintained anonymously.

\section{Acknowledgments}

The authors are glad to thank the MEASURE DHS Project for free access to the Ethiopia Demographic and Health Survey 2016 data.

\section{Consent for publication}

Not applicable.

\section{Disclosure}

The authors declare there was no competing interest. 
Figures

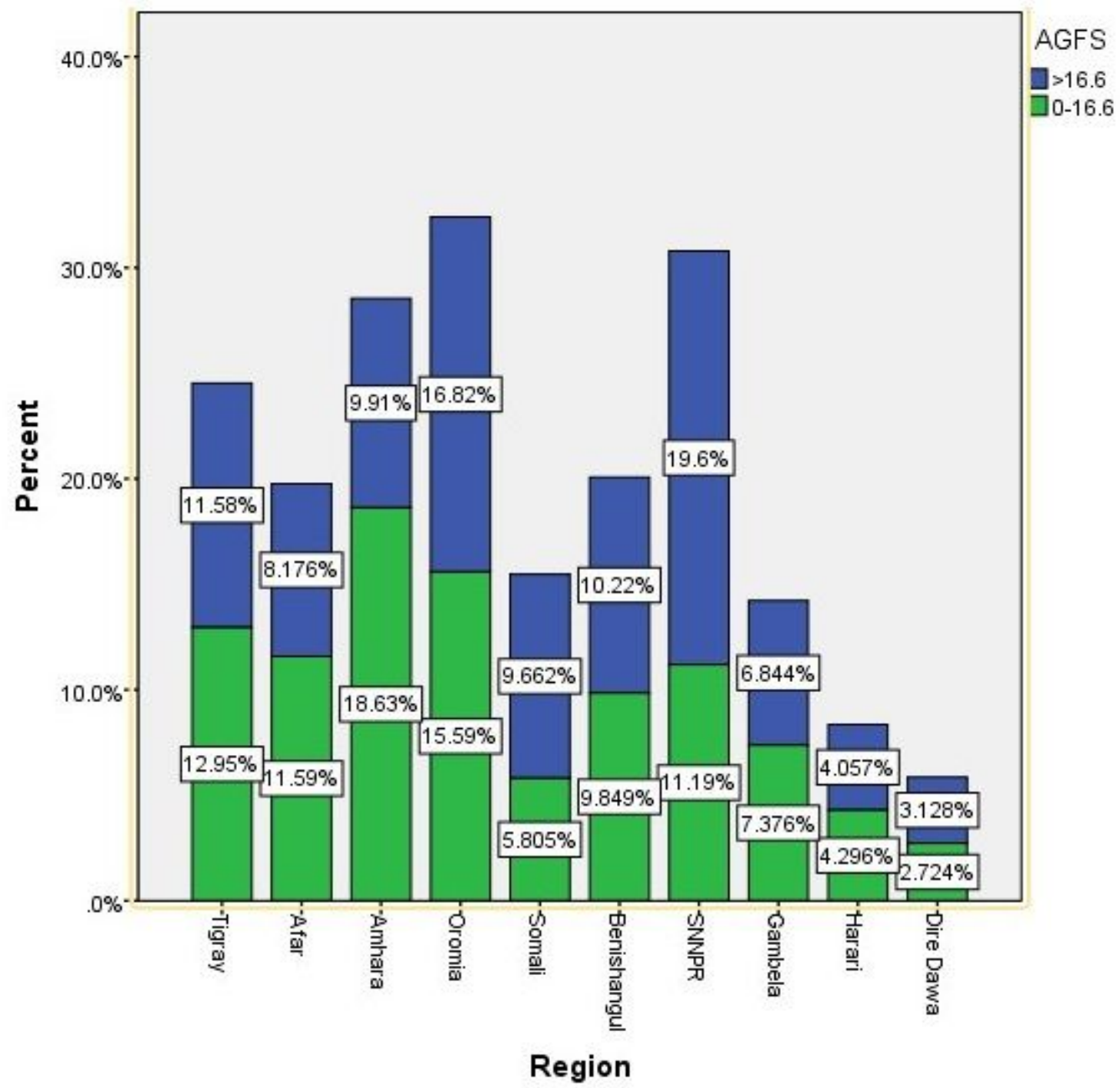

Figure 1

Geographical region on early age sexual initiation in rural Ethiopia, EDHS $2016(n=8001)$ 